\title{
Comparison of Male and Female Rats in Avoidance of a Moving Object: More Thigmotaxis, Hypolocomotion and Fear-Like Reactions in Females
}

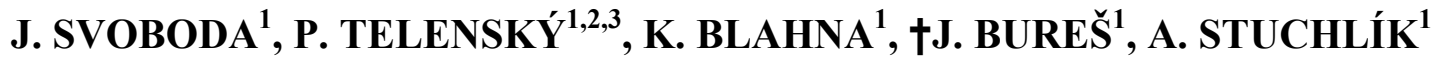 \\ ${ }^{1}$ Institute of Physiology, Academy of Sciences of the Czech Republic, Prague, Czech Republic, \\ ${ }^{2}$ Faculty of Sciences, Charles University, Prague, Czech Republic, ${ }^{3}$ International Clinical Research \\ Center, St. Anne's University Hospital Brno, Czech Republic
}

Received June 11, 2012

Accepted October 15, 2012

On-line October 25, 2012

† Deceased August 24, 2012

\begin{abstract}
Summary
Although male rats generally outperform females in many spatial tasks, sometimes gender differences are not present. This preliminary study examined gender effects in the Enemy avoidance task, in which a rat on a stable circular arena avoids approaching a small mobile robot while collecting randomly dispersed small pellets. Whenever distance between robot and the rat dropped below $25 \mathrm{~cm}$, animal was punished by a mild footshock. Female rats showed thigmotaxis, hypolocomotion and avoidance of robot in the habituation phase, when approaches were not punished. No statistically significant differences in avoidance learning under reinforcement training sessions were observed; but females still spent significantly more time at periphery of the arena and foraged less than males. We conclude that females were able to perform at the same level as males under reinforcement despite different behavioral strategy. The thigmotaxic behavior appears to function as innate escape strategy in female rats triggered by the stressing effect of the moving robot rather then the presence of shocks.
\end{abstract}

\section{Key words}

Gender differences • Enemy avoidance task • Strategies • Anxiety - Thigmotaxis

\section{Corresponding author}

A. Stuchlík, Institute of Physiology Academy of Sciences of the Czech Republic, Vídeňská 1083, 14220 Prague 4, Czech Republic. Fax: +420 24106 2488. E-mail: stuchlik@biomed.cas.cz
Human males usually outperform females in spatial tasks (Grön et al. 2000, Cánovas et al. 2011). Similar picture is seen in rats. Female rats perform worse in the Morris water maze (MMW) and radial maze (Roof 1993). Moreover, male rats have relatively larger hippocampus (Diamond 1987), a structure playing a pivotal role in spatial learning. Although the results on gender effect in spatial tasks in rodents have been recently meta-analyzed (Jonasson 2005) and sex differences confirmed, they can be rather subtle and dependent on various parameters, since there are reports of no sex effects found (Healy et al. 1999), or disappearing after modifying task conditions (Beiko et al. 2004). If animals are habituated for several days before training in the MWM (Perrot-Sinal et al. 1996), females perform similarly as males, suggesting effects of initial stress. Indeed, females have higher levels of corticosterone in the MWM and adrenalectomized females reach the same latencies as control males (Beiko et al. 2004). The stress elimination might account for the results where no gender effects were observed.

Second important issue is use of behavioral strategies. Females tend to display more thigmotaxis (swimming near the wall) in the MWM and this limits their performance unless this behavior is abandoned (Beiko et al. 2004, Harris et al. 2009). Additionally, Roof and Stein (1999) found that females navigate also by proximal extramaze cues (such as an experimenter 
staying at the MWM) more than males. An interesting gender dichotomy was found by Cimadevilla et al. (2000), who trained rats in an active place avoidance task on rotating arena (Bures et al. 1997, Stuchlik and Vales 2006, Stuchlik et al. 2008). Males spent most of time in the quadrant opposite to a to-be-avoided sector, whereas females walked into a zone adjacent clockwise to the sector, suggesting a timing strategy, since this area is temporally furthest from the sector (Cimadevilla et al. 2000).

Aim of this study was to assess gender differences in a recently developed „Enemy avoidance task", a hippocampus-dependent test (Telensky et al. 2011), in which rats, collecting food pellets, avoid a moving robot on a dry arena. This task models avoiding a threatening stimulus and can be viewed as continuous updating of a changing information (Telensky et al. 2011). Avoidance of object moving in an arena has never been compared between sexes, but gender differences in anti-predator behaviors were previously documented (Shepherd et al. 1992, Klein et al. 1994). Exposure to a predator or its odor elicited more pronounced defensive behavior in females than males (Shepherd et al. 1992, Klein et al. 1994). Based on these results, we hypothesized that females would perform better in the Enemy avoidance task.

The subjects were six male and six female LongEvans rats, obtained from the Institute's breeding colony (350-450 g), housed per two or three in Plexiglas cages in an animal room with a constant temperature $\left(21^{\circ} \mathrm{C}\right)$ and 12/12h light/dark cycle. Experiments were conducted in daylight hours. Food was restricted to maintain $85 \%$ of the free-feeding weight; water was freely available. All experiments complied with Animal Protection Code of the CR and EU directive 2010/63/EC. Animals were gently implanted with a hypodermic needle with swirled blunted end, providing purchase for a clip delivering shocks (see later). The apparatus (Telensky et al. 2011) was a circular arena (85 cm in diameter) with grounded floor surrounded by $50-\mathrm{cm}$ violet cardboard. A camera monitored two light-emitting diodes: a smaller, attached by a harness to the rat, and a larger on the robot. $50-\mathrm{Hz}$ samples of both diode positions were stored by custombased software as digital coordinates. The custom-made robot (16 x $15 \times 13 \mathrm{~cm}$; (C) Pavel Jiroutek) moved straightforward (velocity $15 \mathrm{~cm} / \mathrm{s}$ ) until it hit the wall, then it stopped for $15 \mathrm{~s}$, turned by a random angle and went in an opposite direction. Rats received 500-ms shocks whenever their distance from the robot dropped below $25 \mathrm{~cm}$. Constant-current shocks were delivered through a wire and subcutaneous needle on the rat's back. This procedure was previously shown to be safe (Czéh et al. 2001, Stuchlik and Vales 2006). The current was titrated (0.2-1.2 $\mathrm{mA}$ ) to elicit an escape response but prevent freezing. Behavioral sessions lasted $20 \mathrm{~min}$.

The study was divided into two phases. Initially, we pursued 9 sessions of habituation, in which rats searched for barley pellets scattered randomly on the arena with the robot moving (6 males; 6 females). A training phase followed (6 sessions; 6 males and 6 females). We measured total distance in a session (locomotion and foraging), number of time at the periphery (defined as outer annulus occupying $1 / 2$ of the arena surface; measuring thigmotaxis) and number of shocks (reflecting avoidance of a moving stimulus). Data were expressed as means and standard errors of the mean (S.E.M). Statistical evaluation of parameters from the final habituation session was done by Student's $t$-tests. A two-way ANOVA (gender $\mathrm{x}$ sessions) with repeated measures on sessions was used for analysis of the training phase. Newman-Keuls post hoc test was used whenever appropriate. Significance was accepted at $\mathrm{P}<0.05$.

In the final habituation session, mean total distance was $23.96 \pm 6.14 \mathrm{~m}$ in females and $71.85 \pm 8.18 \mathrm{~m}$ in males. A $t$-test revealed a highly significant difference $\mathrm{t}(10)=4.68 ; \mathrm{P}<0.001$ (Fig. 1A). We observed that females spent $81.77 \pm 2.83 \%$ of total time at the periphery compared to $51.59 \pm 6.23 \%$ in males. This difference was also significant $\mathrm{t}(10)=4.43 ; \mathrm{P}<0.01$ (Fig. 1B). Although animals did not receive any shocks in habituation, we computed entrances into a zone around robot ("virtual shocks") during the last habituation session. Mean number of virtual shocks was $64.8 \pm 14.4$ in females and $162.8 \pm 37.3$ in males, again showing a significant difference $\mathrm{t}(10)=2.45 ; \mathrm{P}<0.05$ (Fig. 1C). This suggests that female rats walked less, stayed more at the periphery, and also remained further from the robot when approaches to it were not punished.

During the training phase, the number of shocks dramatically decreased compared to habituation phase. Visual inspection of rats during training showed that, again, females walked less distance, stayed more at the periphery, but avoided the robot with similar efficiency. A two-way ANOVA on the total distance revealed a significant main effect of gender $\mathrm{F}[1,10]=26.54$; $\mathrm{P}<0.001$, sessions $\mathrm{F}[5,50]=2.95 ; \mathrm{P}<0.05$ and a significant interaction $\mathrm{F}[5,50]=2.52 ; \mathrm{P}<0.05$ (Fig. 1D). Similarly, analysis of percentage of the time spent in periphery 
(defined as previously) showed a significant effect of gender $(\mathrm{P}<0.05)$, sessions $(\mathrm{P}<0.05)$, and an interaction $(\mathrm{P}<0.05)$ (Fig. 1E). Analysis of number of shocks showed a highly significant effect of sessions $F(5,50)=6.12$;
$\mathrm{P}<0.001$ but not gender $\mathrm{F}(1,10)=0.26 ; \mathrm{P}>0.05$ (Fig. 1F). No interaction between the factors was revealed $\mathrm{F}(5,50)=0.11 ; \mathrm{P}>0.05$.
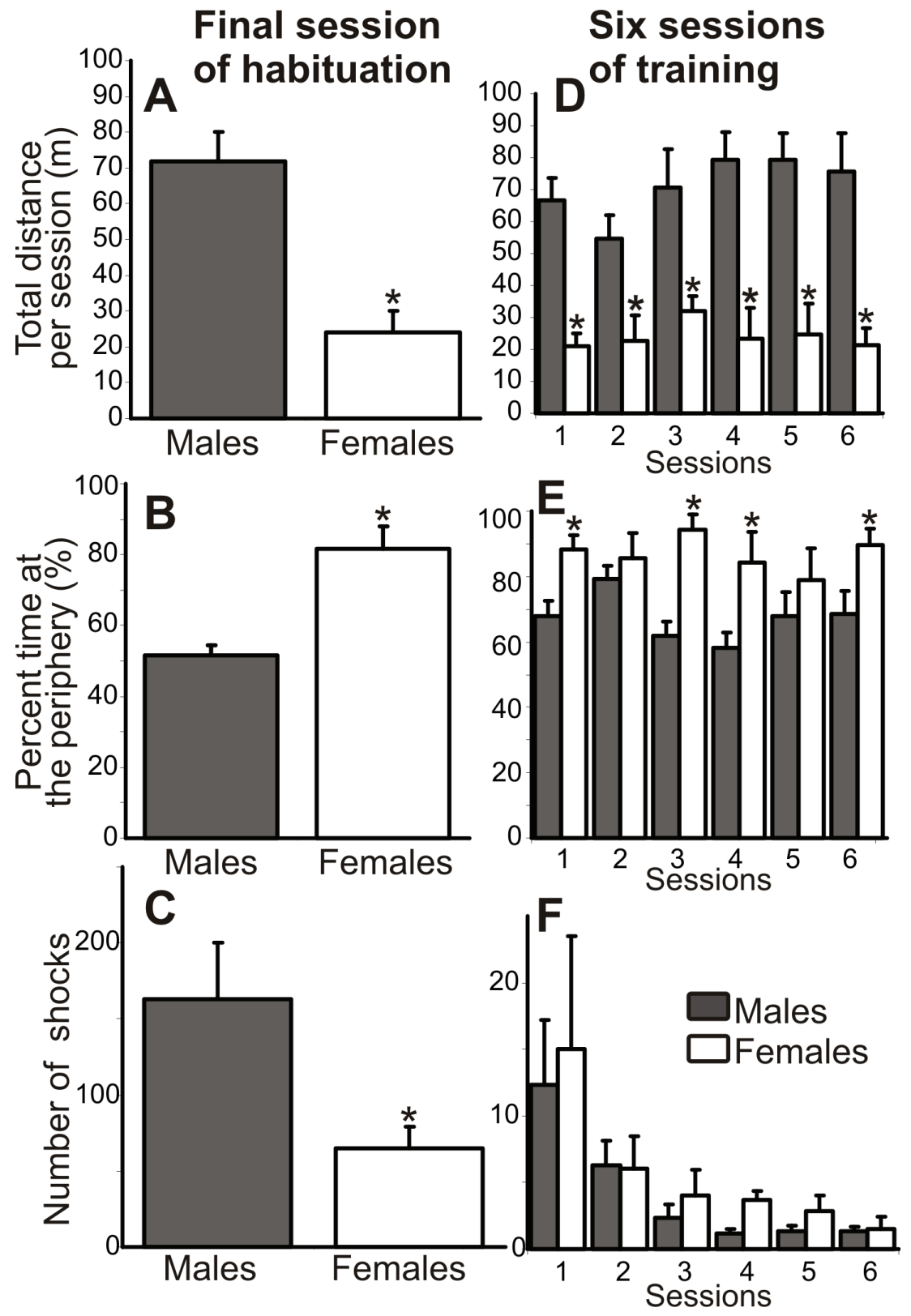

Fig. 1. A. Effect of gender on mean ( \pm S.E.M.) total distance traveled in the final habituation session. Female rats walked significantly shorter paths. B. Thigmotaxis in the final habituation session measured by mean ( \pm S.E.M.) time at the arena periphery (defined as outer annulus occupying one half of the total arena surface) was significantly higher in female rats than male rats. C. Mean ( \pm S.E.M.) number of virtual shocks in the final day of habituation phase. Female rats avoided the robot more then males without a reinforcement. D. Mean $( \pm$ S.E.M.) total distances traveled in particular sessions of the training phase, in which approaches to the robot were punished. Foraging levels were significantly lower in female rats than in male rats. E. Mean ( \pm S.E.M.) time spent at the periphery of the arena was higher in females than males in the training phase. F. Mean ( \pm S.E.M.) number of shocks was relatively low and similar in both genders during training under reinforcement conditions, although there is a trend of impairment in females. $* P<0.05$. Black bars denote males, white bars denote females.
Results of this study showed that females were persistently less active than males in the initial habituation phase, displaying shorter paths, less "virtual shocks" and more thigmotaxis compared to males, which contradicted our expectation that 9-day habituation would suffice to familiarize with the novel environment and eliminate possible initial gender differences in locomotion seen previously in the open-field test (Tropp and Markus 2001). Indeed, our results indicate long- lasting persistence of fear-related thigmotaxis and hypolocomotion, probably due to a presence of the moving robot. Females did not cover the arena uniformly as males to obtain maximum number of pellets and preferred the peripheral part of the arena. Moreover, female paths were three times shorter than in males, reflecting lower foraging and locomotor activity. Notably, female rats seemed to be stressed by the moving robot even when no shocks were given and generated 
fear-related withdrawal behavior, suggesting higher levels of stress. These results are supported by previous findings showing superior defensive reaction to a predator or its odor in female compared to male rats (Shepherd et al. 1992, Klein et al. 1994). However, we cannot conclude that females already avoided robot in the habituation phase since their decreased encountering the robot might be simply accounted by reduced locomotion.

During training phase; however, no statistically significant gender difference was found in learning to avoid approaching the robot (which was punished), although males and females used evidently different strategies. However, a detailed look on the Figure $1 \mathrm{~F}$ suggests that in some sessions there was a trend worse performance in females. In the final session, nonetheless, the performance was almost equal. This may also indicate that female rats would be transiently impaired relative to males, but if they receive enough training, eventually they attain the same level of performance. Nonetheless, this issue requires further detailed study. The difference in strategies is again in accordance with Shepherd et al. (1992), who reported females to produce more intense fear-like responses when confronted with a predator. Our results; however, conclusively show that actual avoidance of a robot (measured by number of shocks), which is dependent upon hippocampus (Telensky et al. 2011), is similar in both genders, showing that both males and females perform with the same efficiency under reinforcement conditions. We therefore cannot support the idea that fear-eliciting stimulus compromises spatial learning in female rats as observed in MWM (Mazor et al. 2009). We suggest that water maze is more sensitive to gender-related differences due to higher demands on spatial cognition and more variability in terms of strategies used to solve the task. For example, females may tend to use different set of landmarks to locate the platform (Roof and Stein 1999). In our task, the only relevant cue is the robot itself, making the solution more straightforward yet hippocampus-dependent (Telensky et al. 2011).

The present study has two major limitations. The first is a relatively low sample size (groups of six animals) and the second is the absence of observation of the estrous cycle of female rats and its relation to the behavioral data. However, since this study presents preliminary data, a more detailed study of defensive reactions in rats to a mobile object including effects of detection of somatic parameters and neocortical lesions is currently being planned.

To summarize, female rats tended to stay and walk more at the wall of the apparatus, had lower locomotion, which led to approaching the robot less when no shocks were given. Under aversive reinforcement, thigmotaxic and hypolocomotion strategy of female rats persisted, but statistically was as efficient in avoiding the moving object as that of male rats. Thigmotaxis therefore did not appear to be mere result of shocking itself; but likely reflected the presence of the mobile object. Next studies in Enemy avoidance tasks are under development in the laboratory.

\section{Conflict of Interest}

There is no conflict of interest.

\section{Acknowledgements}

This study was supported by GACR (P303/10/J032, P304/12/G069), by IGA MZCR NT13386, by AVCRM200111204 and by institutional support (RVO 67985823, AV0Z50110509). We thank Pavel Jiroutek for construction of the robot and technicians of the laboratory for their support. We dedicated this work to Dr. Jan Bureš, DSc., who was our modest but prominent mentor and his legacy will never be forgotten.

\section{References}

BEIKO J, LANDER R, HAMPSON E, BOON F, CAIN DP: Contribution of sex differences in the acute stress response to sex differences in water maze performance in the rat. Behav Brain Res 151: 239-253, 2004.

BURES J, FENTON AA, KAMINSKY Y, ZINYUK L: Place cells and place navigation. Proc Natl Acad Sci USA 94: 343-350, 1997.

CIMADEVILLA JM, FENTON AA, BURES J: Continuous place avoidance task reveals differences in spatial navigation in male and female rats. Behav Brain Res 107: 161-169, 2000.

CZÉH B, STUCHLIK A, WESIERSKA M, CIMADEVILLA JM, POKORNÝ J, SERESS L, BURES J: Effect of neonatal dentate gyrus lesion on allothetic and idiothetic navigation in rats. Neurobiol Learn Mem 75: 190-213, 2001. 
CÁNOVAS R, GARCÍA RF, CIMADEVILLA JM: Effect of reference frames and number of cues available on the spatial orientation of males and females in a virtual memory task. Behav Brain Res 216: 116-121, 2011.

DIAMOND MC: Sex differences in the rat forebrain. Brain Res 434: 235-240, 1987.

GRÖN G, WUNDERLICH AP, SPITZER M, TOMCZAK R, RIEPE MW: Brain activation during human navigation: gender-different neural networks as substrate of performance. Nat Neurosci 3: 404-408, 2000.

HARRIS AP, D'EEATH RB, HEALY SD: Environmental enrichment enhances spatial cognition in rats by reducing thigmotaxis (wall hugging) during testing. Anim Behav 77: 1459-1464, 2009.

HEALY SD, BRAHAM SR, BRAITHWAITE VA: Spatial working memory in rats: no differences between the sexes. Proc Biol Sci 266: 2303-2308, 1999.

JONASSON Z: Meta-analysis of sex differences in rodent models of learning and memory: a review of behavioral and biological data. Neurosci Biobehav Rev 28: 811-825, 2005.

KLEIN SL, LAMBERT KG, DURR D, SCHAEFER T, WARING RE: Influence of environmental enrichment and sex on predator stress response in rats. Physiol Behav 56: 291-297, 1994.

MAZOR A, MATAR MA, KAPLAN Z, KOZLOVSKY N, ZOHAR J, COHEN H: Gender-related qualitative differences in baseline and post-stress anxiety responses are not reflected in the incidence of criterion-based PTSD-like behaviour patterns. World J Biol Psychiatry 10: 856-869, 2009.

PERROT-SINAL TS, KOSTENUIK MA, OSSENKOPP KP, KAVALIERS M: Sex differences in performance in the Morris water maze and the effects of initial nonstationary hidden platform training. Behav Neurosci 110: 13091320, 1996.

ROOF RL, STEIN DG: Gender differences in Morris water maze performance depend on task parameters. Physiol Behav 68: 81-86, 1999.

ROOF RL: Neonatal exogenous testosterone modifies sex difference in radial arm and Morris water maze performance in prepubescent and adult rats. Behav Brain Res 53: 1-10, 1993.

SHEPHERD JK, FLORES T, RODGERS RJ, BLANCHARD RJ, BLANCHARD DC: The anxiety/defense test battery: influence of gender and ritanserin treatment on antipredator defensive behavior. Physiol Behav 51: 277-285, 1992.

STUCHLIK A, VALES K: Effect of dopamine D1 receptor antagonist SCH23390 and D1 agonist A77636 on active allothetic place avoidance, a spatial cognition task. Behav Brain Res 172: 250-255, 2006.

STUCHLIK A, PETRASEK T, VALES K: Dopamine D2 receptors and alpha1-adrenoceptors synergistically modulate locomotion and behavior of rats in a place avoidance task. Behav Brain Res 189: 139-144, 2006.

TELENSKY P, SVOBODA J, BLAHNA K, BURES J, KUBIK S, STUCHLIK A: Functional inactivation of the rat hippocampus disrupts avoidance of a moving object. Proc Natl Acad Sci USA 108: 5414-5418, 2011.

TROPP J, MARKUS EJ: Sex differences in the dynamics of cue utilization and exploratory behavior. Behav Brain Res 119: 143-154, 2001. 